\title{
A Cross Layer Solution for Better Interactions Between Routing and Transport Protocols in MANET
}

\author{
Sofiane Hamrioui ${ }^{1,2}$, Pascal Lorenz ${ }^{1}$, Jaime Lloret $^{3}$ and Mustapha Lalam ${ }^{2}$ \\ ${ }^{1}$ GRTC, UHA University, France \\ 2 UMMTO and USTHB Universities, Algeria \\ 3 Universidad Politecnica de Valencia, Spain
}

This paper presents firstly a study of interactions between two routing protocols (AODV, DSR) and two transport protocol versions (TCP New Reno, Vegas). After this study, we conduct some simulations to analyze the behavior of these protocols with different node mobility and network load values. The performance results of each protocol are examined to identify our own scenarios in order to evaluate our proposed solution for better interactions between these protocols. This solution is called CL-TCP (Cross Layer TCP). It is an adaptation of the TCP congestion control mechanism with some parameters provided by the routing layer (mobility nodes and the size of routing length). After the evaluation of CL-TCP, the results showed that it significantly improves the interactions between TCP Vegas and AODV protocols.

Keywords: MANET, transport protocol, routing protocol, cross layer solution, performance improvement

\section{Introduction}

The performance evaluation of MANETs has become a very important area. In the related literature we can find several studies about MANET network and transport layers. A MANET (Basagni et al., 2004) is a set of mobile terminals independent from any infrastructure, communicating by using radio waves, wherein each terminal can provide a relay service by accepting even the messages which are not intended for it in order to reissue them to another terminal in the network. MANET can be used in any application where the deployment of a wired network infrastructure is too restrictive and difficult to install (Garcia et al. 2008).
Routing function (Mohapatra and Krishnamurthy, 2005) is a delivery method of information between two communicating mobiles through a given connection network. It consists of a strategy which guarantees at any time, a correct and effective establishment of routes between any pair of nodes in the network, which ensures the exchange of messages in a continuous way. The most important problem in MANET is how to adapt this method to a large number of mobile nodes because they have modest computing power and memory (Tarique et al. 2010).

TCP protocol (Holland and Vaidya, 1999) (Hanbali et $a l ., 2005$ ) is currently one of the main protocols used in Internet, more than $80 \%$ of wired communications use it. It seems then natural to use it for reliable communications which involve a wireless link. Unfortunately, TCP is originally developed for fixed networks and is not suited to the characteristics of wireless links. For this reason, TCP suffers from several limitations which tend to grow especially when the interactions between this protocol and the other layers are not taken into account. The study of such interactions and its optimization for better performance of MANET is the aim of our present paper.

Our work is the continuation of those done in (Hamrioui et al., 2012a) and (Hamrioui et al., 2012b). In (Hamrioui et al., 2012a), we used the routing level to improve the energy consumption in the network. We proposed a new approach to minimize the energy consumption called EM-OLSR (Energy Efficiency in MANET by improving OLSR protocol). EM- 
OLSR is based on the OLSR routing protocol and adds a new energy fairness parameter to the Multi-Point Relay (MPR) technique. This new parameter is used by EM-OLSR and allows fairness energy consumption in the same set of MPR. In (Hamrioui et al., 2012b), we used the interactions between the MAC and routing layers to improve the energy consumption in the network. We proposed a new approach called IMR-EE (Improvement of the Interactions between MAC and Routing protocol for Energy Efficient). IMR-EE exploits tow communication environment parameters which are the number of nodes and their mobility. However, in our present paper we use a different type of the interaction to improve the performance of another QoS parameter. We exploit the routing and transport levels to propose our improvement for better TCP protocol performance.

After studying the interactions between two routing protocols (AODV, DSR) and two TCP versions (NewReno, Vegas), CL-TCP (Cross Layer TCP) solution is proposed to improve these interactions. CL-TCP proposes an adaptation of the congestion control mechanism of TCP with some information provided from the routing layer and focusing on route length (in terms of number of hops) and the mobility of nodes. CL-TCP will then be simulated and evaluated using NS-2 simulator according to some performance parameters which are the throughput and the end-to-end delay.

The remainder of the paper is as follows. After a short presentation of the studied protocols in Section 2, we give the most significant works and approaches proposed for better interactions between transport and routing protocols in MANET in Section 3. Then, in Section 4, we turn to the presentation of our CL-TCP solution and show its implementation in NS-2 in Section 5. Next, in Section 6, we study the impact of CL-TCP on the network performance, particularly on throughput and the end-to-end delay. Finally, Section 7 provides our conclusion and future work.

\section{Brief Overview of the Studied Protocols}

TCP is a transport protocol that provides connection-oriented service to upper layers (session, presentation and application). It provides reliable service and is therefore used in end-to-end packet communications between two computers. Several variants of this protocol have been proposed to better adapt it to wireless networks, essentially TCP NewReno and TCP Vegas.

TCP New Reno (Floyd and Henderson, 1999) is an improvement of TCP Reno, it makes changes in Fast Retransmit/Fast Recovery phase. It is used only if the SACK option is not negotiated during the establishment of the connection between the end nodes. Its aim is to overcome the problem of TCP Reno, which is its inability to detect multiple losses simultaneously and therefore avoid the unnecessarily reduction of its congestion window (CWND).

Another version of TCP called TCP Vegas (Oliveira and Braun, 2004) was proposed after some modifications in the source side of Reno. The amendments focus on how to predict the available bandwidth and loss detection. This new algorithm, according to its authors, is able to interact with any valid implementation of TCP.

Routing layer is responsible for forwarding the information to the correct destination through a given wireless connection. Several routing protocols have been proposed in the literature, but only some are suitable for MANETs. In this work, we will study AODV and DSR protocols.

The AODV protocol (Ad hoc On Demand Distance Vector) (Perkins et al., 2006) is a routing protocol designed by Charles E. Perkins and Elizabeth M. Royer and specified in RFC 3561. It is a reactive protocol based on the distance vectors principle. This protocol uses two mechanisms "route discovery" and "route maintenance" and builds the routes by using request cycle "route request/route reply".

DSR (Dynamic Source Routing) (Johnson et al., 2007) was developed by the monarch project at Carnegie Mellon University. It is a reactive and unicast routing protocol, simple and effective, which is dedicated to multihop mobile adhoc networks. It is based on the use of the "source routing" technique. It computes correct routes in the presence of asymmetric links (unidirectional). In this protocol, the loop routing problem is avoided.

\section{Related Work}

In (Bakalis and Lawal, 2010), the authors conducted some simulations to evaluate the performance of CBR over TCP on MANET using 
DSR routing protocol. Although CBR and TCP affect MANET significantly, these differences led to obtaining significant performance results of CBR over TCP with better throughput and less average maximum end-to-end delay. DSR was able to respond to link failure at low pause time, which led to the improvement of TCP's performance in packets delivery. They conclude that TCP traffic models can be used for small network where frequent topology changes are limited and could be controlled by DSR protocol.

In (Mohd Zaini et al., 2012), the authors studied the behavior of TCP Friendly Rate Control (TFRC) and TCP in the presence of DSR and AODV as routing protocols. The used evaluation parameters are throughput, delay and jitter. Their study also allowed them to identify which routing protocols have an impact on transport protocols. They showed that the rate of change of throughput between routing protocols is 3.74.

In (Papanastasiou et al., 2006), the authors present an analysis of TCP performance (including throughput) during the break route events. Three routing protocols are employed, AODV, DSR and OLSR, to better understand the different types of TCP behavior during these events while highlighting the mechanisms that affect each protocol. Their study showed that DSR interacts better with TCP protocol than the others which happens because of its quick routes restoration. AODV showed a good performance avoiding the RTO by caching TCP packets exiting when there is a link failure. For OLSR, by default it maintains settings under the optimal value for the studied scenario as if it were used for traffic loads across loaded topologies.

In (Chaudhary and Singh, 2012) the authors conducted an investigation of the performance of some routing protocols in the presence of $\mathrm{CBR}$ and TCP traffic sources. Two reactive protocols (AODV, DSR) and one proactive (DSDV) were used. Their results showed that reactive protocols have better performance with CBR than with TCP in terms of throughput, delay and packet loss. Moreover, reactive protocols are able to respond quickly to broken links, thus avoiding congestion.

In (Rahman et al., 2008) the authors investigate the performance of TCP over DSDV (proactive) and AODV (reactive) protocols using simulations in $n s-2$ for a range of node mobility with a single traffic source. They found that the proactive protocol consumes more bandwidth, because it transmits routing updates frequently. It reacts slowly in dynamic topologies. Its performance decreases drastically as mobility increases. But the reactive one consumes less bandwidth and lower overhead of routing information. They concluded that to resist against the performance degradation of TCP under high mobility, it is necessary to have some sort of feedback from link layer protocol.

In (Appaji and Sreedhar, 2012) the authors improved transmission performance by distinguishing between packet loss due to link failure and arbitrary loss of packets. They introduced two algorithms: Congestion State Prediction Algorithm (CSPA) and Group Outlet Directive Algorithm (GODA). CSPA helps to distinguish between packet loss due to link failure and arbitrary packet loss. Once the congestion contention node is found, GODA attempts to resolve it at the source node and identifies it as a victim of congestion. If congestion is not resolved at node level, it attempts to handle at group level, and this process continues at predecessor groups if it fails to control congestion at current group level.

In (Hamrioui et al., 2012a), the author proposes a new approach to minimise the energy consumption called EM-OLSR (Energy Efficiency in MANET by improving OLSR proto$\mathrm{col})$. EM-OLSR is based on the OLSR routing protocol and adds a new energy fairness parameter to the Multi-Point Relay (MPR) technique. This new parameter is used in our approach and allows fairness energy consumption in the same set of MPR. In this mechanism, nodes with low power are prevented in the routing process in order to maintain similar power values for all the mobile nodes. The simulation results showed that our proposed EM-OLSR approach allows significant power saving up to $14 \%$ and an increase in average lifetime of a mobile node as high as $22 \%$.

In (Hamrioui et al., 2012b) the authors present an improvement for the interactions between MAC and routing protocols to better energy consumption in MANET (Mobile Ad hoc Networks) and show its incidences on the performance of the network. They propose a new approach called IMR-EE (Improvement of the Interactions between MAC and Routing protocol for Energy Efficient) which exploits tow communication environment parameters. The first one is the number of nodes; our approach reduces the additional energy used to transmit 
the lost data by making the size of the backoff interval of MAC protocol adaptable to the nodes number in the network. The second parameter is the mobility of nodes; IMR-EE uses also the mobility of nodes to calculate the fairness threshold in order to guarantee the same level of the residual energy for each node in the network.

In conclusion, none of the previous studies has addressed all or even some of the parameters of the communication involved in the degradation of the interactions between routing and transport protocols. Some works have only studied the parameters related to the network traffic. In fact, modeling of these parameters and their behaviors in an approach to provide better interactions between the communication protocols will be a great contribution to MANET performance. Our work focuses precisely on these TCP parameters in order to provide an efficient solution in terms of performance. By exploiting some information provided at routing level we show that it is possible to ensure better throughput and the end-to-end delay performance.

\section{Interactions between Routing and Transport Protocols}

\subsection{Evaluation Environment}

The performance evaluation can be done using three techniques: analytical methods, real measurements, and, finally, the simulation. The use of simulation has many advantages, it is faster, less resource consuming, repeatable and can isolate parameters that can sometimes affect the performance. In addition, there are scenarios that are very difficult to be studied in the real world.

The evaluation is carried through the simulation environment NS-2 (version 2.34). The implementation of AODV and DSR protocols is integrated in NS-2. At MAC level, the model $802.11 \mathrm{~b}$ is used by keeping the default values of the parameters of this model.

Some values, such as the duration of simulation, the speed of nodes and the number of connections have been configured properly in order to obtain interpretable results like those reported in the literature. The simulations are performed for 1000 seconds. Nodes were moving in an area of $1000 \mathrm{~m} \times 1000 \mathrm{~m}$. Each node had a transmission range of $250 \mathrm{~m}$. We chose 1000 seconds in order to analyze the interaction of TCP with the routing alternatives in the simulation environment.

The choice of the AODV and DSR routing protocols is justified by the fact that, although both are on demand routing protocols, each one has its own mechanism and is totally different from the other. For transport layer, we opted for TCP New Reno and TCP Vegas. TCP New Reno is a reactive variant. It is widely deployed, and its performance was evaluated in conditions similar to those conducted here. TCP Vegas is a proactive transport protocol with particular features and mechanisms completely different from TCP New Reno. TCP traffic has been used as the main network traffic.

This study is far from being exhaustive. It presents the results using just one mobility model: Random Waypoint (Hyytiä and Virtamo, 2005). However, we have chosen it because the network is not designed for particular mobility and this model is widely used in the related literature. In this model, the mobility of the nodes is typically random and all nodes are uniformly distributed in the simulation space. In this model, some mobile nodes are placed in an area where they cannot leave. An initial position, speed and destination are assigned to each mobile node. Whenever the mobile nodes reach their destination within the surface, they leave to another randomly chosen destination after an optional resting period.

\subsection{Evaluation Parameters}

Since most works published in the related literature, and presented in the previous section, analyzed the throughput and the end-to-end delay parameters, we decided to analyze the same in our study too. The throughput is given by the received data ratio taking into account all data sent. The second parameter is the end-to-end delay which is given by the time a packet takes to travel across the network from a source to a destination. 


\subsection{Effect of Mobility on the Intercactions between Routing and TCP Protocols}

Because our interest focuses on the interaction between routing and transport protocols in MANET, it is clear that the first evaluation should be the mobility performance. In this section we consider 20 different mobility scenarios for each outcome. The scenarios consist of 50 nodes moving at a speed between 0 and $40 \mathrm{~m} / \mathrm{s}$ according to the Random Waypoint mobility model with a time pause of 5 seconds. Ten TCP connections are established between randomly selected pairs.

As it is shown in Figure 1, independently of the speed, it is clear that DSR records better throughput than AODV for TCP New Reno. It should be noted that for low speeds the throughput increases, but it goes down as soon as the speed increases and this happens with both routing protocols. However, an increase was again recorded for both protocols when interacting with New Reno from the speeds exceeding $35 \mathrm{~m} / \mathrm{s}$.

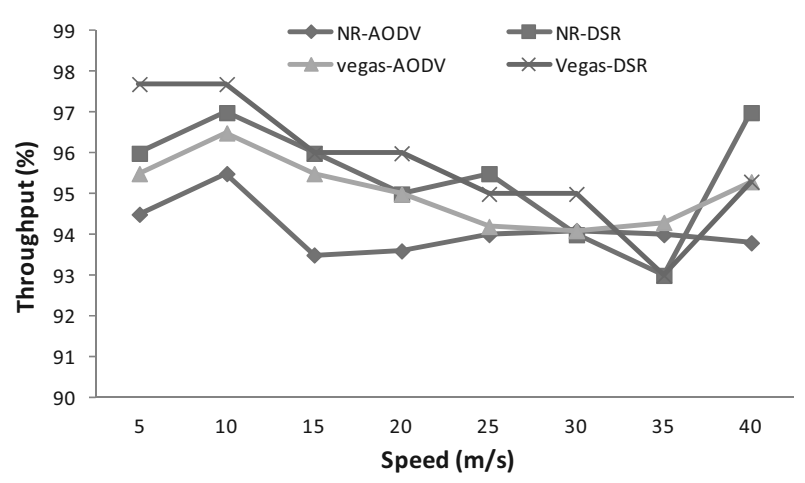

Figure 1. Effect of the mobility on the throughput.

Initially, the throughput increases because the number of received packets increases, but increasing speed causes more broken links and therefore transmission failures and packet loss which again degrade the throughput. The problems of route failures in such environments lead to the activation of a congestion control system which reduces the congestion window and then the throughput is reduced.

It is obvious that the restoration of routes due to link failures caused by the mobility is heavy because both routing protocols are reactive. However, DSR recorded a poor performance because of the invalid routes in the cache after changing the topology and therefore the need for an alternative route or discovering a new one.

The throughput of TCP Vegas in both routing protocols shows an increase for low speeds but this increase is decreased when increasing the speed of the nodes.

For strong mobility, link breaks and node disconnections make packet losses. With these losses, the congestion control system is activated and it reduces the congestion window. However, this difference recorded between both routing protocols is mainly due to the principle of rehabilitation of routes used by every node and it allows a better throughput for TCP Vegas with DSR, although the correct routing is provided by AODV because its recovery mechanism is more effective in this environment. However, the throughput of DSR decreases until it is lower than AODV. DSR throughput increases again from speed $=25 \mathrm{~m} / \mathrm{s}$, but is still lower than AODV.

Generally, we can say that the good performance of DSR is quickly deteriorated due to mobility.

Figure 2 shows that New Reno records better end-to-end delay with AODV than with DSR. Certainly, DSR has the caching mechanism which reduces the routing overhead at intermediate nodes. However, the available routes should be still valid. Mobility implies topology changes and generally broken links which leads to route failures and packet losses.

However, a very interesting point must be raised, at certain speeds the time may decrease, which means that mobility can also be beneficial.

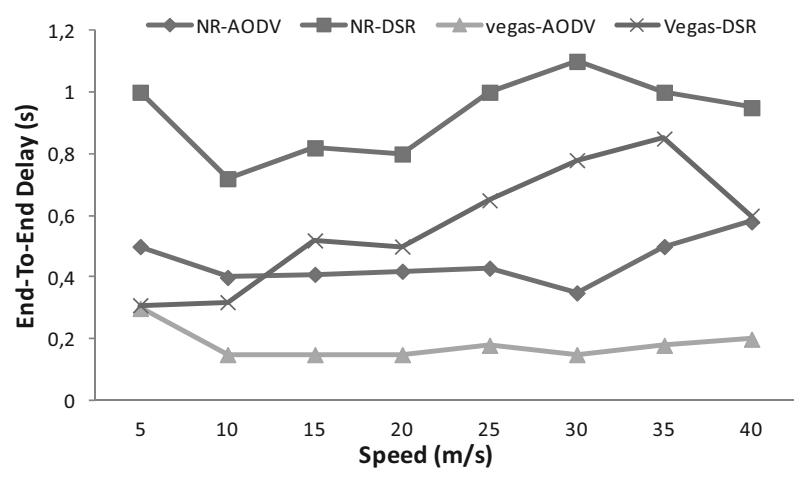

Figure 2. Effect of the mobility on the end-to-end delay. 
A result that has been observed in simulations is that the end-to-end delay of TPC Vegas with DSR at low speeds is very similar to AODV and sometimes it can be better. This is due to the rapid restoration of routes from the caches of the intermediate nodes.

The average end-to-end delay of TPC Vegas recorded with AODV remains much lower, compared to that recorded with DSR when the speed increases. The delay for DSR increases rapidly with the mobility increase. In fact, when mobility increases AODV is better, compared to DSR. The unstable routes are the origin of this degradation.

\subsection{Effect of Network Load}

In the second step, we made an evaluation varying the size of the network. The scenarios considered in this section consist of nodes moving at a speed of $15 \mathrm{~m} / \mathrm{s}$ according to the Random Waypoint mobility model, with a pause time of 5 seconds. Ten TCP connections were established between randomly selected pairs. An average of twenty different scenarios was conducted for each outcome. The number of nodes is varied between 20 and 100 nodes.

In Figure 3 we can see that in a network with a large amount of numbers of nodes TCP Vegas and TCP New Reno recorded a degradation of the throughput, particularly with the DSR protocol. The difference between both recorded throughputs is due to the principle of routes rehabilitation used by every node. For a few numbers of nodes, DSR records better throughput with both TCP versions because its cache mechanism reduces the routing overhead at intermediate nodes in order to get better throughput. However, for large values of the numbers of nodes, DSR, even with its mechanism, recorded a poor performance because of invalid routes in the cache after changing the network load and therefore the need of an alternative route or discovering a new one.

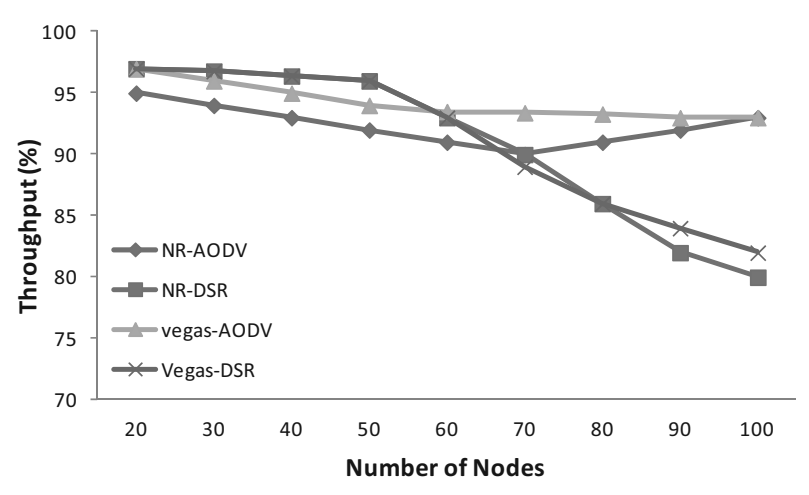

Figure 3. Effect of the network load on the throughput.

Figure 4 shows that in large size networks the end-to-end delay of a TCP Reno new packet is much better with DSR than with AODV, due to the overhead generated by the field path. In addition, the non negligible probability of having an invalid route in the cache explains the higher end-to-end delay of DSR, compared to AODV. TCP Vegas recorded higher average end-to-end delay with DSR, compared to AODV, even for smaller number of nodes.

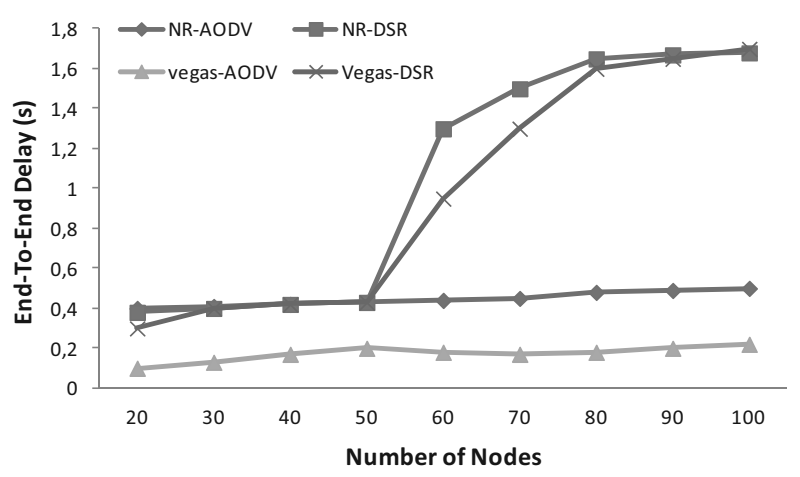

Figure 4. Effect of the mobility on the end-to-end delay.

After the previous study, we have seen that the interactions between protocols are influenced by the communication environment parameters studied: the mobility and network load. It constitutes the basis of the evaluation of our solution, through which we select the worst results of throughput and end-to-end delay. The corresponding scenarios will be used to evaluate the performance of our proposed improvement. Finally, a new performance comparison will be made, which will include the empirical results identified previously and those obtained after implementing our solution. 


\section{Improvement of Interactions between Routing and Transport Protocols}

We have already seen that the performance of TCP is strongly influenced by the network layer. TCP Vegas, as proactive transport protocol, shows good performance under different conditions. However, it provides much better results with AODV routing protocol than with DSR. For this reason, AODV and TCP Vegas will be used for our proposed optimizations.

Our proposed solution CL-TCP uses the transport layer and gives more consideration to some information gathered from the routing layer that helps TCP to know the reasons of packet loss and reduces the number of cases of unnecessary initiation of the congestion control mechanism.

The first part of our proposed CL-TCP considers the mobility of the nodes. In fact, node mobility often leads to the breakdown of connectivity between nodes, resulting in data loss. These data losses may, although it is not always the case, be interpreted by the transport protocol as losses due to congestion. Then, it activates the congestion control mechanism which will reduce the unnecessarily throughput. For this reason, our solution also uses another threshold to guarantee the same level of the residual energy for each node in the network.

For this reason, TCP-CL takes into account the information of node mobility, which is received from the routing layer. As we know, the mobility is generally characterized by its speed and angle of movement. These two factors determine the degree of the impact of mobility on packet loss.

In order to model our system, we consider a node $i$, in communication with another node $j$, then we note:

$\alpha_{i, j}$ : the angle between the line $(i, j)$ and the movement direction of node $i$,

$W_{i}$ : the speed of mobile node $i$.

We have seen in the previous study that for very small speeds, TCP performance parameters are not affected, and larger values of mobility can also help to improve performance in the case where the communicating node doesn't move far from its neighbouring node (with whom he communicates). Based on that, we proceed with the development of the first part of CL-TCP.
Let $H\left(W_{i}\right)$ and $G\left(A_{i, j}\right)$ be two logical functions whose values are determined as follows:

$$
H\left(W_{i}\right)= \begin{cases}\text { true } & \text { if } S_{\text {min }}<W_{i}<S_{\text {max }} \\ \text { false } & \text { else }\end{cases}
$$

Here, $S_{\text {min }}$ is the speed of node $i$ from which network performance begins to degrade. Its value is determined by the mobility model. In our case, it will be fixed at $10 \mathrm{~m} / \mathrm{s} . S_{\max }$ is the speed of node $i$ from which network performance begins to grow again. These two values must be determined according to the mobility model. In our case, it will be fixed at $35 \mathrm{~m} / \mathrm{s}$ (through our previous results).

$G\left(\alpha_{i, j}\right)$ is another logic function which relates to the angle $\alpha_{i, j}$ and informs TC-TCP if the direction of the movement of node $i$ can lead to packet loss due to a broken link. Its value is determined as follows:

$$
G\left(\alpha_{i, j}\right)= \begin{cases}\text { false } & \text { if }-\pi / 4 \leq \alpha_{i, j} \leq \pi / 4 \\ \text { true } & \text { else }\end{cases}
$$

CL-TCP allows each node $i$ to get the value of its current $\mathrm{Si}$. The easiest way to do it is to deduce it by knowing the time spent between two geographical points. There are many systems for node mobile location such as GPS and power measurement techniques (Elliott, 2005) (Doherty et al., 2001). With these systems, each node can know its position at any time, and then, it will be able to estimate the distance travelled during an interval of time. With the distance and time we can get the speed of the mobility $W_{i}$. Moreover, it is possible to determine the direction of their movements and the value of the angle $\alpha_{i, j}$.

CL-TCP also uses information relating to the number of nodes that contain the routing path. We have shown in (Hamrioui et al., 2007) (Hamrioui and Lalam, 2008), slightly delaying acknowledgment well received TCP packets can improve the performance of the protocol. We apply the same principle here, but instead of the number of nodes, we will use the time required for one packet to move between the source and destination. Let $T_{r}$ be this time, each node $i$ after received $T_{r}$ will run the following treatment:

$$
T_{r}:=T_{r}+T_{i, i+1}
$$

Here $T_{i, i+1}$ is the time elapsed during the packet transmission from node $i$ to the next node in the routing path, the node $i+1$. 
Next two pseudo algorithms show how our proposed improvements are used by the CL-TCP protocol.

\section{For routing side:}

$\{$ The previous code of $C L-T C P\}$

\section{Begin}

$\mid \operatorname{Rup}_{1}$, Rup $_{2}:=$ False

| For $i:=1$ to $N-1$ do $\{N$ is the number of nodes in the network\}

|| $T_{r}:=T_{r}+T_{i, i+1}$

\section{| End For}

Send $\left(T_{r}\right)$ \{send () is function which send information from routing layer to transport layer $\}$

| While (rup1=False) and $(i \leq N)$ do

|| $\operatorname{Rup}_{1}:=H\left(W_{i}\right) ; \operatorname{Rep}_{2}:=\mathrm{G}\left(\alpha_{i, j}\right) ; i:=i+1$

\section{| End While}

| If non (Rep or Rrep $\left._{2}\right)$ then

|| $i:=1, \|$ While $\left(\right.$ Rup $_{1}=$ False $)$ and $(i \leq N)$ do

$\| \operatorname{Rep}_{1}:=F(W i) ; i:=i+1$

\section{| End While}

\section{| End If}

| Send $\left(\operatorname{Rup}_{1}, \operatorname{Rup}_{2}, T_{r}\right)$

\section{End}

The following code of $C L-T C P\}$

\section{For transport side:}

$\{$ The previous code of $C L-T C P\}$

\section{Begin}

| Inform $\left(N_{s}, N_{d}\right) ;\left\{N_{s}\right.$ is the source node, $N_{d}$ is the destination node $\}$

| If $R T O<T_{r}$ Then

|| $R T O:=\left(R T O+T_{r}\right) / 2$

\section{| End If}

| If congestion is detected Then

|| If $\left(\right.$ Rup $_{1}$ or Rup $\left._{2}\right)$ Then

|| Ignore the congestion

\section{| End If}

| End If

\section{End}

\{The following code of CL-TCP

\section{Incidences of the Improvement of Interactions between Routing and Transport Protocols}

In this section, we evaluate our proposed solution by showing its impact on the performance of MANET, especially on two parameters previously studied (throughput and end-to-end delay). To do this, we kept the same simulation environment. Moreover, the same evaluation parameters are used. The results are compared to those obtained with AODV routing protocol and TCP Vegas version. This choice is justified by the fact that in our previous study, these two protocols have allowed us to obtain the best results in terms of throughput and end-to-end delay.

\subsection{Effect of the Mobility}

Figure 5 shows that our TCP-CL solution presents an improvement of TCP throughput. In fact, the two optimizations made for both AODV and TCP Vegas protocols allowed it to better manage the packet loss. When mobility increases, the probability of having more broken links becomes important which leads to many packet losses in the network. CL-TCP informs TCP about the real reason of these losses, which allows it to avoid initiating unnecessarily the congestion control mechanism. This explains maintaining of better throughput although a slight decrease was recorded. CLTCP therefore allows both AODV and TCP Vegas protocols to interact and better understand the sources of packet losses in the network.

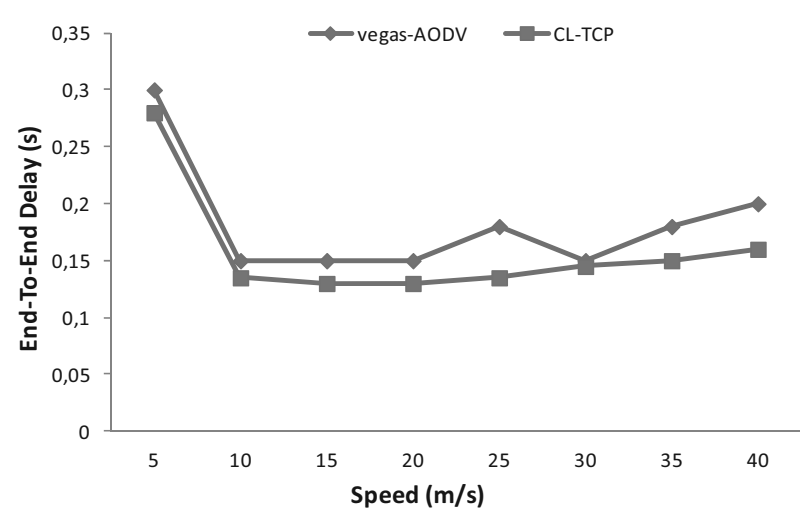

Figure 5. Effect of the mobility on the throughput. 
In Figure 6 we note that our solution also provides an improvement of end-to-end delay. In fact, with CL-TCP, the congestion control mechanism is not triggered unnecessarily after packet losses due to the mobility. Therefore, the transmission time of packets is minimized which leads to the improvement of the end-to-end delay. Moreover, with CL-TCP, an optimal time to wait for TCP packets acknowledgment (RTO) is negotiated between the routing and TCP protocols. The obtained time takes in consideration the new routes formed by the routing protocol after breaking the connectivity which can be short (in this case, the RTO must be reduced). With this optimization of time, CL-TCP improves the end-to-end delay.

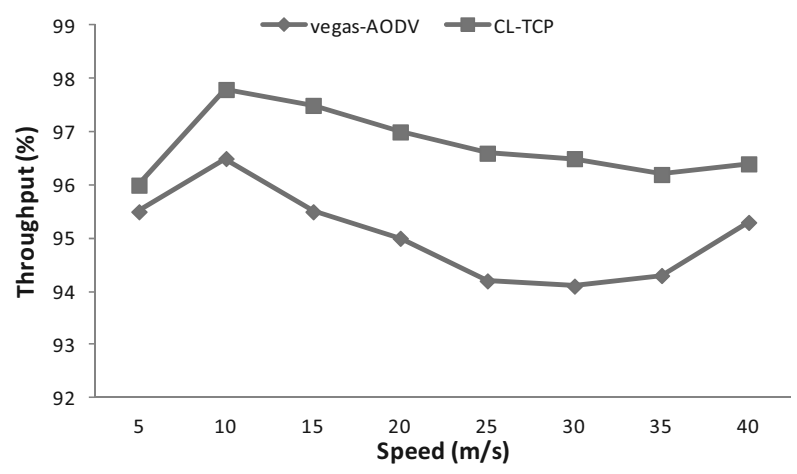

Figure 6. Effect of the mobility on the end-to-end delay.

\subsection{Effect of the Network Load}

Figure 7 shows the effect of the number of nodes in the network of interactions between protocols AODV and TCP Vegas before and after the improvement. With the increasing number of nodes, there is a TCP throughput degradation, but this degradation is slight compared to that recorded before the improvement. In fact, TCPCL takes into account the information about the number and the nodes used for the calculation of an optimal waiting time for receiving acknowledgments of TCP packets. With this optimization, retransmissions and unnecessary waiting times are avoided, which allows a greater throughput with our solution CL-TCP.

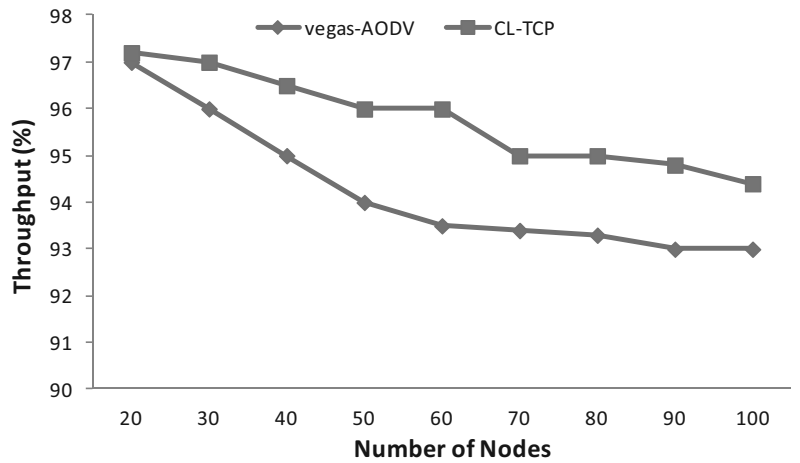

Figure 7. Effect of the network load on the throughput.

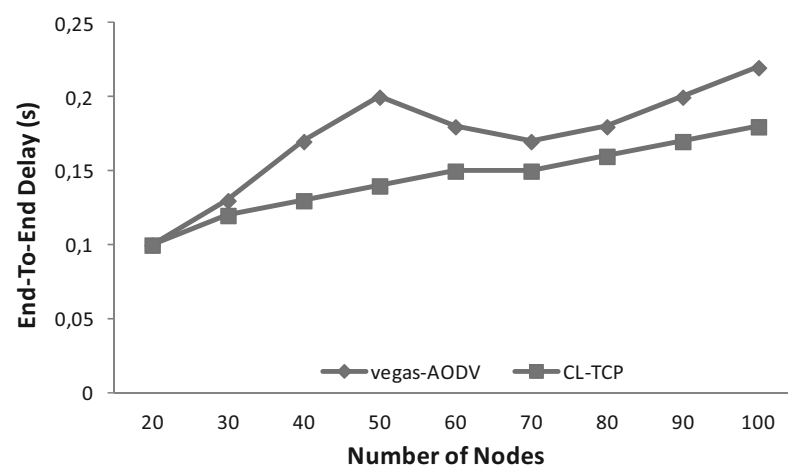

Figure 8. Effect of the network load on the end-to-end delay.

Network load also has a significant effect on the interaction of the two AODV and TCP Vegas protocols. Moreover, the number of nodes increases the end-to-end delay increases too. But, with our solution this increase is lower. In fact with the increase of the number of nodes, the routes created and used by the routing protocol can become important (in terms of number of nodes contained in the routing path), which makes the time to go and get back across the route more important. CL-TCP negotiates the waiting time for TCP acknowledgments by taking into account the routes length in routing side, which allows TCP to allow the required time to receive the TCP acknowledgments before knowing that its corresponding data packets are lost. Therefore, the additional time for the retransmission of the supposed lost TCP packets is avoided, which improves the end-to-end delay. 


\section{Conclusion and Perspectives}

In this paper, after studying the interactions between two routing protocols (AODV and DSR) and two TCP versions (NewReno and Vegas), CL-TCP (Cross Layer TCP) solution is proposed to improve these interactions. CL-TCP proposes an adaptation of the TCP congestion control mechanism with some information provided from the routing layer and focusing on the route length (in terms of number of hops) and the mobility of nodes.

After the implementation and simulation of CLTCP, we studied its incidences on the MANET performance, more particularly on some performance parameters which are the throughput and the end-to-end delay of the TCP protocol. The obtained results are very conclusive and satisfactory: CL-TCP helps the transport layer to distinguish the lost packets due to the congestion from those packets lost because of the load nodes and their mobility. With this improvement, there is a significant increase of the performance of TCP in terms of throughput and end-to-end delay.

In our future work, we will continue modeling the maximum number of communication environment parameters. We will try to reflect the communication environment as much as possible. Our CL-TCP solution will be compared with other solutions proposed in the same context and they will be tested on a real platform; in this case, we really need to produce all the phenomena existing in a real MANET.

\section{References}

[1] A. Hanbali, E. Altman, P. Nain, A Survey of TCP over Ad Hoc Networks. IEEE Communications Surveys \& Tutorials, 7(3) (2005), pp. 22-36.

[2] S. BASAgni, M. Conti, S. Giordano, I. StojmenOVIC, Mobile Ad hoc Networking. Wiley-IEEE Press, USA, 2004.

[3] C. E. Perkins, E. M. Royer, S. R. DAs, Ad Hoc OnDemand Distance-Vector (AODV) Routing. IETF Internet draft (draft-ietf-manet-aodv-o6.txt).

[4] D. Johnson, Y. Hu, D. Maltz, The Dynamic Source Routing Protocol (DSR) for Mobile Ad Hoc Networks for IPv4, RFC4728, The IETF Trust (2007)
[5] D. K. ElLIOTT, Understanding GPS: Principles and Applications. Second Edition. Artech House Publishers, (2005).

[6] E. Hyytia, J. VitTAmo, Randomwaypoint model in n-dimensional space. Operations Research Letters, 33 (2005), 567-571.

[7] G. Holland, N. H. Vaidya, Analysis of TCP performance over mobile ad hoc networks. Seattle, August 1999. Annual International Conference on Mobile Computing and Networking (Mobicom'99).

[8] K. Mohd Zaini, A. M. M. Habbal, F. AzZali, S. HASSAN, M. RIZAL, An Interaction between Congestion-Control Based Transport Protocols and Manet Routing Protocols. Journal of Computer Science, 8(4) (2012), 468-473.

[9] L. Doherty, K. S. J. Pister, L. El GhaOuI, Convex position estimation in wireless sensor networks. In Proc. of the IEEE INFOCOM, (2001), vol. 3, pp. 1655-1663, Alaska.

[10] M. Garcia, H. COll, D. BRI, J. Lloret, Using MANET protocols in Wireless Sensor and Actor Networks. The Second International Conference on Sensor Technologies and Applications (SENSORCOMM 2008), August 25-31, (2008), Cap Esterel, (France).

[11] M. M. Rahman, M. A. NAShiRy, T. K. Godder, S. ALI, TCP Performance over Proactive \& Reactive Routing Protocols for Mobile Ad Hoc Network. Accepted in the Proceedings of International Conference of Wireless Communication (ICWN), July 14-17, (2008), Las Vegas, Nevada, USA.

[12] M. S. Chaudhary, V. Singh, Simulation and analysis of routing protocol under CBR and TCP traffic source. International Conference on Communication and Network Technologies, IEEE, (2012).

[13] M. Tarique, A. Hossain, R. Islam, C. A. HosSAIN, Issues of Long-Hop and Short-Hop Routing in Mobile Ad Hoc Networks: A Comprehensive Study. Network Protocols and Algorithms, 2(2) (2010), pp. 107-131.

[14] NS2. NETWORK SIMULATOR. http://www.isi.edu/nsnam

[15] P. BAKALIS, B. LAWAL, Performance Evaluation of CBR and TCP Traffic Models on MANET Using DSR Routing Protocol. CMC'10 Proceedings of the 2010 International Conference on Communications and Mobile Computing, vol. 03, 318-322.

[16] P. MOHAPATRA, S. V. KRISHNAMURTHY, Ad hoc Networks Technologies and Protocols. Springer Science + Business Media, Inc, 2005, eBook ISBN: 0-38722690-7, Print ISBN: 0-387-22689-3.

[17] R. OliveIRA, T. Braun, A Dynamic Adaptive Acknowledgment Strategy for TCP over Multihop Wireless Networks. Proc. IEEE INFOCOM, (2005).

[18] S. Floyd, T. Henderson, New Reno Modification to TCP's Fast Recovery. RFC 2582, 1999. 
[19] S. Hamrioui, M. DaOUi, L. ChameK, M. Lalam, P. LORENZ, Incidences of the Improvement of the Interactions Between MAC and Routing Protocols on MANET Performance. Journal of Advanced Computer Science and Technology, 1(4) (2012), 250-265, Science Publishing Corporation, ISSN: 2227-4332 (online), ISSN: 2227-4839.

[20] S. HAMRIOUI, M. LALAM, Incidence of the Improvement of the Transport - MAC Protocols Interactions on MANET Performance. $8^{\text {th }}$ Annual International Conference on New Technologies of Distributed Systems (NOTERE 2008), (2008), France.

[21] S. HAMrioui, M. LALAM, Incidences of the Improvement of the MAC-Transport and MACRouting Interactions on MANET Performance. International Conference on Next Generation Networks and Services, (2010), Morocco.

[22] S. HAMrioui, M. LALAM, P. LOREnZ, A new approach for energy efficient in MANET based on the OLSR protocol. International Journal of Wireless and Mobile Computing, 5(3) (2012), 292-299, Inderscience Publishers, Issn (online): 1741-1092, Issn (print): 1741-1084.

[23] S. Papanastasiou, L. M. Machenzie, M. OuldKHAOUAN, V. CHARISSIS, On the interaction of TCP and Routing Protocols in MANETs. Advanced International Conference on Telecommunication and International Conference on Internet and Web Applications and Services, IEEE, (2006).

[24] V. V. APPAJI, M. SReEdHAR, CRT: Crosslayered Routing Topology for Congestion Control in Mobile Ad Hoc Networks. International Journal of Scientific \& Engineering Research, Volume 3, Issue 9, September-2012 2 ISSN 2229-5518.

Received: February, 2013

Revised: May, 2013

Accepted: August, 2013

Contact addresses:

Sofiane Hamrioui GRTC

University of Haute Alsace (UHA)

Colmar

France

e-mail: s.hamrioui@gmail.com

Pascal Lorenz GRTC

University of Haute Alsace (UHA)

Colmar

France

e-mail: Lorenz@ieee.org

Jaime LIoret

Universidad Politecnica de Valencia

Spain

e-mail: jlloret@dcom.upv.es

Mustapha Lalam

LARI

Mouloud Mammeri University (UMMTO)

Tizi Ouzou

Algeria
SOFIANE HAMRIOUI received the engineering degree in computer science option parallel and distributed systems in 2004 and the master degree in computer science in 2007 from the Mouloud Mammeri University of Tizi Ouzou, Algeria. Since 2008, he has been teacher at the Department of Computer Science, University of Science and Technology Houari Boumediene, Algiers, Algeria. He is enrolled in doctoral thesis since 2008 and it looks as part of its research are issues in mobile and ad hoc wireless networks as well as interactions between protocols, performance protocols, quality of services and saving energy.

PASCAL LORENZ received a PhD degree from the University of Nancy, France. Between 1990 and 1995 he was a research engineer at WorldFIP Europe and at Alcatel-Alsthom. He is a professor at the University of Haute Alsace and responsible for the Network and Telecommunication Research Group. His research interests include QoS, wireless networks and high-speed networks. He is senior member of the IEEE, member of many international program committees and he has served as a guest editor for a number of journals including Telecommunications Systems, IEEE communications Magazine and LNCS.

JAIME LIORET received his M.Sc. in Physics in 1997 from the University of Valencia and a postgraduate Master in Corporative Networks and Systems Integration from the Department of Communications of the Polytechnic University of Valencia in 1999. He received his M.Sc. in Electronic Engineering in 2003 from the University of Valencia and his Ph.D. in Telecommunication Engineering (Dr. Ing.) from the Polytechnic University of Valencia in 2006. Until 2008, he had more than 55 scientific papers published in national and international conferences, more than 25 educational papers and more than 25 papers published in international journals (several of them with Journal Citation Report).

MUSTAPHA LALAM received the Master's degree in Computer Architecture from the High School of Computer Science, Algiers, Algeria, in 1980 and the $\mathrm{PhD}$ degree in Computer Science from University of Toulouse, France, in 1990. He joined the University of Tizi Ouzou, Algeria in 1993, where he is now Professor in the Computer Science Department at the University of Tizi Ouzou. He has been involved in research and development of computer architecture, distributed systems and mobility management for wireless mobile computing and communications. 